\title{
Swarm intelligence for a single source product distribution
}

\section{Surafel Luleseged Tilahun}

Department of Mathematics,

Addis Ababa Science and Technology University,

Addis Ababa, Ethiopia

Email: surafe142@gmail.com

\begin{abstract}
Distributing products under different constraints is one of the challenging task in industries. In this paper a single-source resource distribution to different centres using finite and small number of transportation system is discussed. For a production centre as the source, there could be multiple centres to transport the products using trucks and each truck should have equal load in terms of centres which they are going to distribute the products to. The problem can be seen as a set of multiple travelling salesman problem. This problem will be formulated and a custom-made swarm intelligence algorithm based on prey-predator algorithm will be used. Three datasets of different categories, including cases when there is a restriction to go from one centre to another, are generated and used to test the algorithm. The datasets are also attached in the Appendix for future research and comparison.
\end{abstract}

Keywords: swarm intelligence applications; product distribution; travelling salesman problem; prey-predator algorithm.

Reference to this paper should be made as follows: Tilahun, S.L. (2022) 'Swarm intelligence for a single source product distribution', Int. J. Advanced Intelligence Paradigms, Vol. 21, Nos. 1/2, pp.87-99.

Biographical notes: Surafel Luleseged Tilahun completed his $\mathrm{PhD}$ in Applied and Computational Mathematics from the Universiti Sains Malaysia, Malaysia in 2013. He also completed his MSc in Computational Science, MSc in Mathematics and BSc in Mathematics from the Addis Ababa University, Ethiopia in 2009, 2007 and 2004, respectively. He was a postdoctoral researcher in the School of Mathematics, Statistics and Computer Science, University of KwaZulu-Natal, South Africa. Currently, he is an Associate Professor in the Department of Mathematics, Addis Ababa Science and technology University, Ethiopia. His research interest is in general on applied and computation mathematics focusing in computational optimisation, decision making and artificial intelligence.

\section{Introduction}

The application and uses optimisation techniques become very important and crucial for different application including industrial production and distribution applications. Different industries deal with the concept of optimisation or decision making in different stage of 
their production and also in the distribution of produced goods (Liu and Papageorgiou, 2013; Andres et al., 2017; Ariafar et al., 2014). Even though there are different case-based analysis and method used for the production stage, the distribution of goods is sometimes done with a certain familiar routine. Even though different researches are conducted regarding distribution, scheduling and vehicle routing problems they are case-based and based on some assumptions (Ali et al., 2016; Sarmiento and Nagi, 1999; Sarathambekai and Umamaheswari, 2017; Saravanan and Venkatachalam, 2016).

Different decision making problems can be formulated as a mathematical optimisation problem (Tilahun and Asfaw, 2012; Marler and Arora, 2004; Tilahun and Ong, 2012; Firoze and Rahman, 2017). A mathematical optimisation problem is a problem of optimising, which can be either maximising or minimising, a given objective by identifying the best value for the decision variable from the set of feasible candidate solutions. Finding the maximum or minimum of a function is an old but active problem where different researchers have explored. In general, solution methods for an optimisation problem can be categorised as deterministic and non-deterministic methods (Tilahun et al., 2017b; Tilahun and Ngnotchouye, 2017). Metaheuristic algorithms are non-deterministic approaches which are not affected much by the problem property and try to find an approximate solution with proper parameter tuning and sufficient iteration runs (Tilahun, 2017).

Swarm intelligence algorithms are of the second class, i.e., metaheuristic, inspired by collective social behaviour of animals including bees, ants, flocking of birds and schooling of fishes (Lim and Jain, 2009). These algorithms work by exchanging information regarding the landscape of the decision space and guiding the search according to their collective experience during the search. Introducing new, and hybridising existing algorithms is one of the active research area researchers are exploring (Ong et al., 2017). Often combining multiple methods results a superior results as the weakness of one algorithm is compensated by the strength of the other. The theoretical analysis including convergence, asymptotic analysis and also modifying algorithms to boost their performance is also a crucial part of current research community (Yang, 2011). Designing a custom made algorithm for specific problems is also another interesting area where many challenging problems are addressed with (Bindiya and Elias, 2014). Currently, there are hundreds of metaheuristic algorithms with different formulation and inspiration (Rajpurohit et al., 2017).

In this paper the production distribution of a single industry products, source, under limited transportation access will be formulated as a mathematical optimisation problem. Prey-predator algorithm will be used to solve the formulated problem. The algorithm is one of the swarm intelligence algorithm inspired by the interaction between a predator and its prey (Tilahun and Ong, 2015; Hamadneh et al., 2013). It has been tested and found to be effective in a number of applications (Tilahun et al., 2017a; Tilahun, 2012; Bahmani-Firouzi et al., 2015; Dai et al., 2015; Tilahun and Ngnotchouye, 2016a, 2016b). It is a generalised case where some well-known algorithms fall as its special case (Ong et al., 2017). Furthermore, the algorithm is shown to be effective when compared with other algorithms and a number of modifications has been done to boost its performance (Tilahun and Ong, 2013; Tilahun, 2013; Tilahun et al., 2016; Tilahun and Ngnotchouye, 2016c; Tilahun and Matadi, 2018). Since, the algorithm is proposed for continuous optimisation problem, its discrete version which suite the special formulation of the problem will be proposed. A dataset to test the approach will be generated randomly. Hence, in the next section the problem formulation will be discussed followed by a discussion and modification of the algorithm in Section 3. Section 4 discusses the numerical experiment part followed by a discussion in Section 5. Finally, a conclusion will be given in Section 6 . 


\section{Problem formulation}

A single source, industry, product distribution is where there is an industry plant where products are produced and distributed to different centres. In addition, we are assuming that there is a fixed number of means of transportation, without loss of generality assume the means of transportation is trucks. Consider the location of the industry or source be $X_{0}$ and there are $n$ centres where the products needs to be distributed to with equal level of urgency. In addition, consider there are $m$ means of transportation or trucks. If $m \geq n$, then the worst case scenario where each truck will go to one centre can be a possible solution. However, when $m$ is smaller than $n$ then scheduling the trucks becomes a problem. The objective of our model is to minimise the cost (it can also be traveling distance) with the aim of delivering the product to each of the centres. We assume that there is no capacity limitation in the trucks, i.e., a truck can provide necessary amount of products to the centres it visits.

Let a truck be represented with $T_{i}$ for $i \in\{1,2, \ldots, m\}$ and let the centres be numbered from 1 up to $n$. For each track we need to determine a sequence of centres hence that will be represented by the decision variable. Hence, the dimension of the decision variable for each truck varies. Suppose a truck $T_{i}$ passes through $k_{i}$ centres, hence the corresponding route of the car can be $p_{i}=X_{0}-x_{i, 1}-x_{i, 2}-\cdots-x_{i, k_{i}}-X_{0}$ where $x_{i, j} \neq x_{p, q}$ if $i \neq$ $p$ and $j \neq q$. Hence, we need to determine these paths or $x_{i, j}$ 's to minimise the cost or distance travel. Therefore the objective function for each path $p_{i}$ can be represented by $c_{i}=$ $c\left(X_{0}, x(i, 1)\right)+c\left(x\left(i, k_{i}\right), X_{0}\right)+\sum_{j=1}^{k_{i}-1} c\left(x_{i, j}, x_{i, j+1}\right)$. From this the objective function of the problem will be $f(x)=\sum_{i=1}^{m} c_{i}$.

The complete problem can be given as in equation (1).

$$
\begin{aligned}
\min _{x} f(x) & =\sum_{i=1}^{m} c_{i} \\
\text { s.t. } & x_{i, j} \in\{1,2, \ldots, n\} \\
& x_{i, j} \neq x_{p, q} \text { for } i \neq p \wedge j \neq q
\end{aligned}
$$

Additional restrictions in the constraint set like $k_{i}=k_{j}, \forall i, j$ can also be set to simplify the problem. That is each truck should visit equal number of centres before returning to the industry site.

The formulated problem can be considered as $m$ travelling salesman problem. Each of these problems have a variable number of cities to visit. 


\section{Solution approach}

\subsection{Prey-predator algorithm}

Prey-predator algorithm is a swarm-based algorithm (Tilahun and Ong, 2015; Hamadneh et al., 2013). The algorithm is inspired by the relation between a predator and its prey. The algorithm works by starting with initial multiple solutions, at least three, and try to improve their performance by exploring as well as exploiting the solution space. After measuring the performance of the solutions based on the objective function, the solutions will be categorised into three categories, namely the predator, best prey and ordinary prey. A predator is a solution with the least performance of all the solution in that particular iteration, whereas the best prey is a solution with the best performance of all the solution in that particular iteration. The rest of the solutions will be called and their search behaviour will be affected by these two extreme solutions. The predator is assumed to be in a non-promising area and try to explore other parts of the feasible region by running around and at the same time scaring the ordinary prey to increase the exploration property of the algorithm. That is done by having a long jump and also moving towards the weak ordinary prey, weak refers to the performance of the solution according to the objective function. The updating equation of the predator $\left(x_{p}\right)$ is given in equation (2).

$$
x_{p}:=x_{p}+\lambda_{\max } u_{r}+\lambda_{\min }\left(x^{\prime}-x_{p}\right)
$$

where $x^{\prime}$ is the weak prey, $\lambda_{\max }$ and $\lambda_{\min }$ are the exploration and exploitation step length, i.e., $\lambda_{\max }>\lambda_{\min }$. Unlike the predator the best prey totally focuses on exploitation of its neighborhood, as it is assumed to be in the promising region. Hence, it is updated by choosing the best direction among $q$ random directions, where $q$ being an algorithm parameter. If there is no direction among these directions which will improve the solution then the solution will not be updated. The updating equation is given in equation (3)

$$
x_{b}:=x_{b}+\lambda_{\min } u
$$

where $u=\operatorname{argmin}\left\{f\left(x_{b}+\lambda_{\min } u\right) \mid u \in\left\{u_{1}, u_{2}, \ldots, u_{q}, \overrightarrow{0}\right\}\right\}$ for a zero vector $\overrightarrow{0}$.

An ordinary prey $x_{i}$ is either affected by the predator or the best prey based on an algorithm parameter called probability of followup, $P_{f}$. If a random number between zero and one from a uniform distribution is less than or equal to the $P_{f}$, then the solution follow better performing solutions while doing a local search. However if the random number is greater the probability of followup it will randomly run away from the predator. The updating equation of an ordinary prey $x_{i}$ is given in equation (4).

$$
x_{i}:==\left\{\begin{array}{l}
x_{i}+\lambda_{\min } u_{r}+\lambda_{\max } u_{r u n}, \quad \text { if } P_{f}>\text { rand } \\
x_{i}+\lambda_{\min } u_{r}+\lambda_{\max }\left(x_{j}-x_{i}\right) \text { otherwise }
\end{array}\right.
$$

where $u_{r}$ is a random direction, $x_{j}$ is better performing solution and $u_{r u n}$ is a random direction away from the predator. $u_{\text {run }}$ can be computed by generating a random direction $u$ and checking either $u$ or $-u$ which will take the solution away from $x_{p}$.

The algorithm is summarised in Table 1. 
Table 1 Prey-predator algorithm $(f(x)$ is the objective function and $S$ is the feasible region

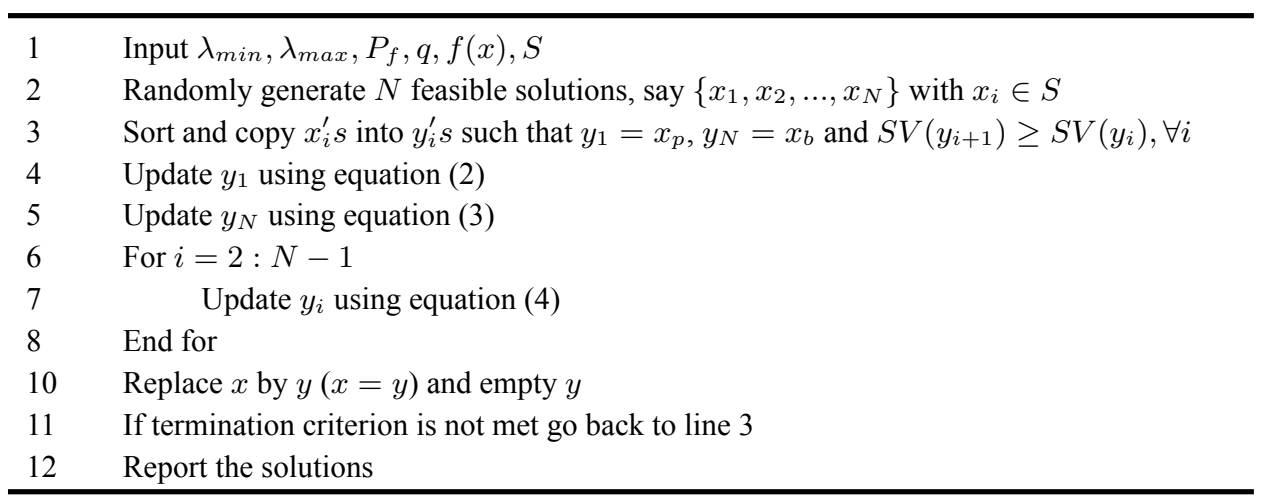

\subsection{The proposed approach}

The standard prey-predator algorithm is designed for a continuous problem and the updating equation will update the solution in a continuous space. Hence, it needs to be modified so that it can be used for our formulated discrete problem. The modified version should be made in such a way that the search behaviour of the algorithm, which is the exploration and exploitation behaviour, is preserved. The algorithm is modified to deal with the optimisation problem given in equation (1) with equal work load in terms of the number of centres visited for each truck, i.e., $k_{i}=k_{j}, \forall i, j$. If the number of the trucks is not a factor of the total number of the centres, dummy centres, whose distance between any other centres and also from the industry is zero, will be added and used. Consider there are $n$ centres and $m$ trucks and assume without loose of generality that $n=r m$ for a positive integer $r$.

Theorem 1: Consider a system with $n$ centres and $m$ means of transportation with equal working load in terms of the centre visiting number. Furthermore, suppose the remainder of $\frac{n}{m}$ is $\bar{r}$ for $\bar{r}<m$. The the solution of a corresponding problem with additional $m-r$ centres of distance zero from the industry as well as the other $n$ centres is a solution for the original problem.

The proof of the theorem is a direct forward. If $x^{*}$ is a solution for the original problem then addition any number of dummy variable which will not contribute to the cost will not change the optimal cost found. However, in the computation of the cost a modification, given as followed, needs to be made. Suppose $x_{d m}$ is the dummy centre variable added. To compute the cost of the routes consider the dummy variable is in between $x_{i}$ and $x_{i+1}$, then the cost to go from $x_{i}$ to $x_{d m}$ is set to be zero, however, the cost from $x_{d m}$ to $x_{i+1}$ should be set to be equal to the cost of going from $x_{i}$ to $x_{i+1}$.

The first step of the algorithm is generating $N$ random feasible solutions. One possible way to construct a feasible solution is making the variable to be a randomly arranged order of the first $n$ positive integers, say $S(n)=S(1), S(2), \ldots, S(n)$, to represent the order of the centres to be visited. Then the route for truck $i$ will be $0, S((i-1) r+1), S((i-1) r+$ $2), \ldots, S(i r), 0$, where 0 is the location of the industry. For example if $n=8$ and $m=3$ then a dummy centre number 9 is added. If the first eight positive integers are arranged 
randomly, say $2,8,1,6,7,3,5,9,4$. Then the route for the first, the second and the third truck will be given by $0,2,8,1,0 ; 0,6,7,3,0$ and $0,5,9,4,0$

The solutions then be evaluated based on the objective function and sorted as $x_{1}, x_{2}, \ldots, x_{N}$, where $x_{1}$ is the predator, $x_{N}$ is the best prey and the rest ordinary prey. That means $f\left(x_{i+1}\right) \leq f\left(x_{i}\right)$, for all $i$.

In the updating stage, unlike the continuous case, the updating will be done component wise and by preserving the feasibility. Hence, the update will be done by changing some of the entries and adjusting the feasibility (that means making sure that there will be no repetition of values). To determine which entries to change algorithm parameter called probability of change is introduced (with the same use as the step lengths). There will be two probability of change, for exploration, $P_{c 1}$ and for exploitation, $P_{c 2}$, with $P_{c 1}>P_{c 2}$. Based on that a predator will be updated according to the following pseudo-code as given in equation (5):

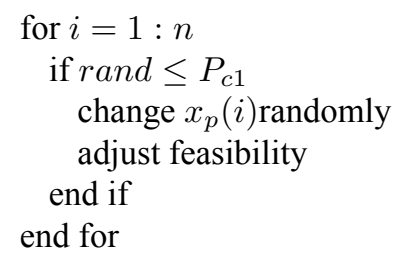

The best prey $x_{b}$ will be updated by generating $q$ solution using equation (5) with $P_{c 1}$ replaced by $P_{c 2}$ and the best solution among these $q$ updated solution will be taken as the updated solution of $x_{b}$.

An ordinary prey, $x_{l}$ will move towards better solution or run away from the predator based on an algorithm parameter called probability of followup, $P_{f}$. If a random number from a uniform distribution between zero and one is less that $P_{f}$, then the solution will follow better performing solutions and otherwise run away from the predator. This movement will be done component wise with rounding and also feasibility adjustment, as given in equation (6).

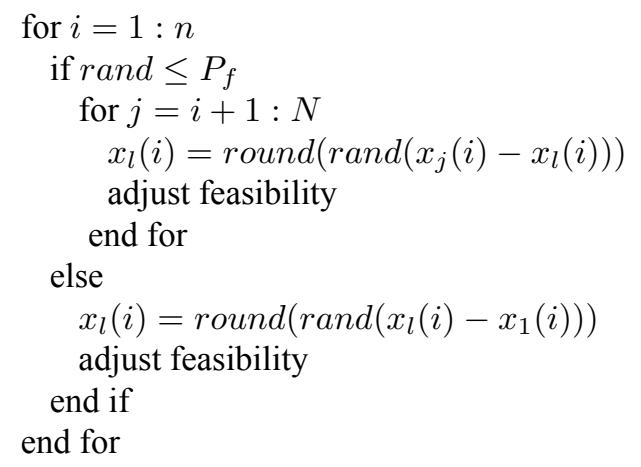

where rand is a random number from a uniform distribution between zero and one and the round function will round up the values to the nearest integer. The feasibility adjustment here make sure that the values are not out of range (greater than $n$ and less than 1 ) and also no repetition in the entries of the vector.

The termination criteria can be either a maximum number of iteration, achieving a certain target level of objective function value or if there is no more improvement in a number of consecutive iterations. 


\section{Numerical discussion}

\subsection{Datasets}

Three random datasets are generated using MATLAB. Basically, a dataset will have a matrix which $n$ by $n$ and a vector of size $n$, where $n$ is the number of centres. The matrix represents the distance between the $n$ centres (i.e., it is a symmetric matrix with diagonal entries zero that is because we are assuming that going from one centre to another has the same distance with returning) and the vector is the distance of the centres from the industry or the source. The entire $d_{i j}$ represents the distance between centre $i$ and $j$ (centre or source). It can also possibly have location of the centres in a coordinate plane with respect to the source which is useful only for visualisation. The dataset is given in the Appendix section.

Dataset 1 The first dataset has ten centres with two trucks. All the centres are connected.

Dataset 2 The second dataset has 11 cities and 3 trucks. Hence a dummy additional centre will be constructed with distance of zero from all the centres and the source. Furthermore, all the cities are connected.

Dataset 3 The third dataset has 16 centres and 4 truck. Furthermore, as can be in the dataset there are no routes between some of the centres, i.e., the entry in the matrix is $\infty$ means there is no route between the corresponding centres.

\subsection{Simulation setup}

MATLAB is used for simulation. The algorithm parameters are set based on recommendations on prey-predator algorithm implementation from the literature (Tilahun, 2017, 2012; Ong et al., 2017; Tilahun and Ong, 2015; Tilahun and Ngnotchouye, 2016b). Accordingly, the probability of followup is set to be 0.75 , probability of change one and two is set to be 0.6 and 0.2 , respectively. The number of random directions $q$ is set to be 20. The termination criteria is taken to be the maximum number of iteration and is set to be 100. The initial number of solutions is taken to be 50 .

The simulation setup including a summary on the datasets is given in Table 2 .

Table 2 Datasets and simulation setup

\begin{tabular}{lccccccccc}
\hline & Connectedness & $\bmod (n, m)=0$ & $n$ & $T$ & $P_{f}$ & $P_{c 1}$ & $P_{c 2}$ & $N$ & $q$ \\
\hline Dataset 1 & Yes & Yes & 10 & 2 & 0.75 & 0.6 & 0.2 & 50 & 20 \\
Dataset 2 & Yes & No & 11 & 3 & 0.75 & 0.6 & 0.2 & 60 & 25 \\
Dataset 1 & No & No & 16 & 4 & 0.75 & 0.6 & 0.2 & 75 & 30 \\
\hline
\end{tabular}

Note: $\bmod (n, m)=0$ means $m$ is a factor of $n$.

\subsection{Simulation results}

After running the algorithm on MATLAB using the discussed simulation setup, the best solution found for the first dataset is $x^{*}=[1,5,7,8,3,4,2,6,9,10]$ with cost of $f(x)=$ 79.9554. That means truck A can start from the industry site go to five centres in the sequence of centre $1,5,7,8$ and 3 before returning to the starting place. Similarly truck B 
will pass through centres 4, 2, 6, 9 and 10 in this given sequence before starting and arriving back from the industry site.

Similarly, $x^{*}=[2,5,6,3,10,4,8,1,12,11,7,9]$ is the final solution found for the second dataset with $f\left(x^{*}\right)=81.4277$. Truck A will go through the industry site and through the four centres in the order of 2, 5, 6 and 3 before returning to the factory. Truck B starts from the factory and go through centres $10,4,8$ and 1 before getting back to the factory. Since centre 12 is a dummy or imaginary centre, truck $\mathrm{C}$ passes through the centres 11,7 and 9 starting and finishing at the industry or factory site.

For the third dataset, $x^{*}=[3,7,12,2,8,11,13,5,10,15,9,16,6,4,1,14]$ with $f\left(x^{*}\right)=89.7054$ is the final solution found. Hence all the four trucks passes through the following routes starting and ending at the industry or factory, for truck A, centres 3, 7, 12, 2; for truck B centres 8, 11, 13, 5; for truck C centres 10,15, 9, 16 and for truck D 6 , $4,1,14$.

The performance of the algorithm for the datasets over the iterations is shown in Figure 1 .

Figure 1 Simulation results, (a) dataset 1 (b) dataset 2 (c) dataset 3 (see online version for colours)

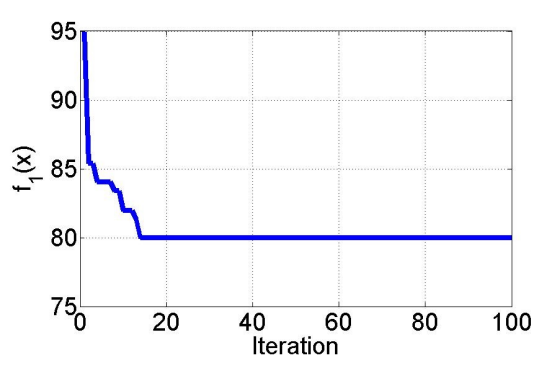

(a)

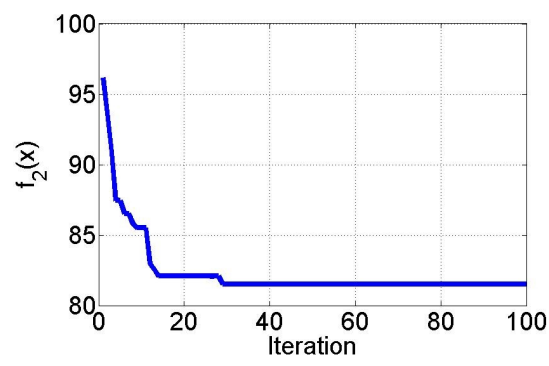

(b)

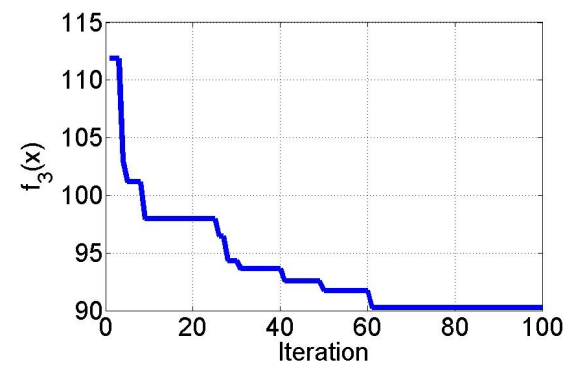

(c)

In addition to a single trial multiple trails were also run to record the average performance of the algorithm over the datasets. The termination criteria also changed from maximum number of iteration to archiving the best solutions found above. with these purposes, the algorithm was run for 30 trials and the resulting solution is summarised and recorded as shown in Table 3. 
Table 3 Simulation results

\begin{tabular}{|c|c|c|c|c|c|c|c|c|}
\hline & \multicolumn{2}{|l|}{ Best } & \multicolumn{2}{|c|}{$f(x)$} & \multicolumn{2}{|c|}{ CPU time } & \multicolumn{2}{|c|}{ Iteration } \\
\hline & $x^{*}$ & $f\left(x^{*}\right)$ & $\mu$ & $S D$ & $\mu$ & $S D$ & $\mu$ & $S D$ \\
\hline Dataset 1 & $\begin{array}{l}{[1,5,7,8,3} \\
\quad 4,2,6,9,10]\end{array}$ & 79.9954 & 79.9954 & 0 & 0.9635 & 0.0152 & 19 & 1.2 \\
\hline Dataset 2 & $\begin{array}{r}{[2,5,6,3,10,4,8} \\
1,12,11,7,9]\end{array}$ & 81.4277 & 81.6307 & 0.2087 & 1.2258 & 0.0028 & 25 & 5.67 \\
\hline Dataset 3 & $\begin{array}{l}{[3,7,12,2,8,11,13,5} \\
10,15,9,16,6,4,1,14]\end{array}$ & 89.7054 & 89.8825 & 0.3067 & 1.8694 & 0.0120 & 63 & 71.25 \\
\hline
\end{tabular}

Note: $x^{*}$ is the best solution found, $\mu$ is the mean and $S D$ is the standard deviation.

\section{Discussion}

A single source product distribution from a factory or production centre to different centres is studied in this paper. The optimisation model of the problem to minimise the distance or cost is formulated. The resulting formulation becomes multiple traveling salesman problem with variable cities or values. The formulation and solution analysis is done based some assumptions, including the means of transportation (trucks) have equal work load with sufficient carrying capacity. That means each truck should visit equal number of centres as much as possible. However, if the number of centres are not equally divided by the trucks, dummy or artificial centres will be introduced to make up the shortage of the centres.

A swarm-based algorithm is introduced based on prey-predator algorithm search paradigm. Random datasets are introduced which includes different scenarios including disconnected centres, unmatched number of centres and trucks. The simulation result shows that the performance of the algorithm improves through iteration. In addition smaller dimensional problems are stable as their standard deviation is smaller.

A number of issues can be considered for future research. One possible issue is when there are multiple sources. There are cases where a factory or industry have multiple production centres. Hence, the study of distribution of products towards minimising cost needs to be studied.

Each truck, in this paper, are sharing (almost) equal working load in terms of the number of centres they visited. This assumption can be relaxed. Hence, different working load in terms of the number of centres a truck visited also needs to be studied.

Testing the approach on high dimensional and highly constrained problems should be done to assess the strength and weakness of the proposed approach. In addition developing well studied set of datasets for comparison purposes needs to be studied, along with proposing other solution approaches and doing a detailed comparison of the methods.

\section{Conclusions}

A single source production distribution problem towards cost minimisation is an applied problem which exist in different industries. A swarm intelligence-based algorithm based on prey-predator algorithm is proposed to solve the problem. The problem is assumed to have equal working load for each truck in terms of number of visiting centres and to meet that dummy or artificial centres may be introduced and used. Three datasets are 
constructed from different problem category including problem involving disconnected cities. Simulation results show that the performance of the algorithm improves with iteration. Future works, including relaxing the assumptions used, is also discussed.

\section{References}

Ali, R., Mounir, G. and Moncef, T. (2016) 'Adaptive probabilities of crossover and mutation in genetic algorithm for solving stochastic vehicle routing problem', International Journal of Advanced Intelligence Paradigms, Vol. 8, No. 3, pp.318-326.

Andres, B., Sanchis, R., Lamothe, J., Saari, L. and Hauser, F. (2017) 'Integrated production-distribution planning optimization models: a review in collaborative networks context', International Journal of Production Management and Engineering, Vol. 5, No. 1, pp.31-38.

Ariafar, S., Ahmed, S., Choudhury, I.A. and Bakar, M.A. (2014) 'Application of fuzzy optimization to production-distribution planning in supply chain management', Mathematical Problems in Engineering, Vol. 2014, Article ID 218132, 8pp [online] https://doi.org/10.1155/2014/218132.

Bahmani-Firouzi, B., Sharifinia, S., Azizipanah-Abarghooee, R. and Niknam, T. (2015) 'Scenario-based optimal bidding strategies of GENCOs in the incomplete information electricity market using a new improved prey-predator optimization algorithm', IEEE Systems Journal, Vol. 9, No. 4, pp.1485-1495.

Bindiya, T.S. and Elias, E. (2014) 'Modified metaheuristic algorithms for the optimal design of multiplier-less non-uniform channel filters', Circuits, Systems, and Signal Processing, Vol. 33, No. 3, pp.815-837.

Dai, W., Liu, Q. and Chai, T.(2015) 'Particle size estimate of grinding processes using random vector functional link networks with improved robustness', Neurocomputing, Vol. 169, pp.361-372 [online] https://doi.org/10.1016/j.neucom.2014.08.098.

Firoze, A. and Rahman, R.M. (2017) 'Critical condition classification of patients from ICCDR, B hospital surveillance data', International Journal of Advanced Intelligence Paradigms, Vol. 9, No. 4, pp.347-369.

Hamadneh, N., Tilahun, S.L., Sathasivam, S. and Choon, O.H. (2013) 'Prey-predator algorithm as a new optimization technique using in radial basis function neural networks', Research Journal of Applied Sciences, Vol. 8, No. 7, pp.383-387.

Lim, C.P. and Jain, L.C. (2009) 'Advances in swarm intelligence', in Innovations in Swarm Intelligence, pp.1-7, Springer Berlin Heidelberg, Berlin .

Liu, S. and Papageorgiou, L.G. (2013) 'Multiobjective optimisation of production, distribution and capacity planning of global supply chains in the process industry', Omega, Vol. 41, No. 2, pp.369-382.

Marler, R.T. and Arora, J.S. (2004) 'Survey of multi-objective optimization methods for engineering', Structural and Multidisciplinary Optimization, Vol. 26, No. 6, p.369-395.

Ong, H.C., Tilahun, S.L., Lee, W.S. and Ngnotchouye, J.M.T. (2017) 'Comparative study of prey-predator algorithm and firefly algorithm', Intelligent Automation and Soft Computing, pp.1-8.

Rajpurohit, J., Sharma, T.K., Abraham, A. and Vaishali, A. (2017) 'Glossary of metaheuristic algorithms', International Journal of Computer Information Systems and Industrial Management Applications, Vol. 9, pp.181-205, ISSN 2150-7988.

Saraswathi, K. and Tamilarasi, A. (2012) 'A modified metaheuristic algorithm for opinion mining', International Journal of Computer Applications, Vol. 58, No. 11, pp.43-47. 
Sarathambekai, S. and Umamaheswari, K. (2017) 'Performance comparison of discrete particle swarm optimisation and shuffled frog leaping algorithm in multiprocessor task scheduling problem', International Journal of Advanced Intelligence Paradigms, Vol. 9, Nos. 2-3, pp.139-163.

Saravanan, S. and Venkatachalam, V. (2016) 'Improving map reduce task scheduling and micro-partitioning mechanism for mobile cloud multimedia services', International Journal of Advanced Intelligence Paradigms, Vol. 8, No. 2, pp.156-167.

Sarmiento, A.M. and Nagi, R. (1999) 'A review of integrated analysis of productiondistribution systems', IIE Transactions, Vol. 31, No. 11, pp.1061-1074.

Tilahun, S.L. and Asfaw, A. (2012) 'Modeling the expansion of Prosopis juliflora and determining its optimum utilization rate to control the invasion in Afar Regional State of Ethiopia', International Journal of Applied Mathematical Research, Vol. 1, No. 4, p.726-743.

Tilahun, S.L. and Matadi, M.B. (2018) 'Weight minimization of a speed reducer using prey-predator algorithm', International Journal of Manufacturing, Materials, and Mechanical Engineering (IJMMME), Vol. 8, No. 2, pp.19-32.

Tilahun, S.L. and Ngnotchouye, J.M.T. (2016a) 'Two stage travel salesman model of world tourism', PRICAI2016 The 14th Pacific Rim International Conference on Artificial Intelligence, AI4T, 22-26 August, Phuket, Thailand.

Tilahun, S.L. and Ngnotchouye, J.M.T. (2016b) 'Examination timetabling using prey-predator algorithm', PRICAI2016 The 14th Pacific Rim International Conference on Artificial Intelligence, AIED, 22-26 August, Phuket, Thailand.

Tilahun, S.L. and Ngnotchouye, J.M.T. (2016c) 'Prey-predator algorithm with adaptive step length', International Journal of Bio-Inspired Computation, Vol. 8, No. 4, pp.195-204.

Tilahun, S.L. and Ngnotchouye, J.M.T. (2017) 'Firefly algorithm for discrete optimization problems: a survey', KSCE Journal of Civil Engineering, Vol. 21, No. 2, pp.535-545.

Tilahun, S.L. and Ong, H.C. (2012) 'Bus timetabling as a fuzzy multiobjective optimization problem using preference-based genetic algorithm', Promet-Traffic and Transportation, Vol. 24, No. 3 , pp.183-191.

Tilahun, S.L. and Ong, H.C. (2013) 'Comparison between genetic algorithm and prey-predator algorithm', Malaysian Journal of Fundamental and Applied Sciences, Vol. 9, No. 4, pp.167-170.

Tilahun, S.L. and Ong, H. C. (2015) 'Prey-predator algorithm: a new metaheuristic algorithm for optimization problems', International Journal of Information Technology and Decision Making, Vol. 14, No. 6, pp.1331-1352.

Tilahun, S.L., Ong, H.C. and Ngnotchouye, J.M.T. (2016) 'Extended prey-predator algorithm with a group hunting scenario', Advances in Operations Research, Vol. 2016, Article ID 7325263, 14pp [online] http://dx.doi.org/10.1155/2016/7325263.

Tilahun, S.L., Goshu, N.N. and Ngnotchouye, J.M.T. (2017a) 'Prey-predator algorithm for travelling salesman problem: application on the ethiopian tourism sites', Handbook of Research on Holistic Optimization Techniques in the Hospitality, Tourism, and Travel Industry, pp.402-422.

Tilahun, S.L., Ngnotchouye, J.M.T. and Hamadneh, N.N. (2017b) 'Continuous versions of firefly algorithm: a review', Artificial Intelligence Review, pp.1-48 [online] https://doi.org/10.1007/s10462-017-9568-0.

Tilahun, S.L. (2012) 'Fuzzy graph representation of bus timetabling problem and its solution method using prey-predator algorithm', inInternational Workshop on Optimal Network Topologies (IWONT).

Tilahun, S.L. (2013) Prey-predator Algorithm: a New Metaheuristic Optimization Approach, PhD thesis, School of Mathematical Sciences, Universiti Sains Malaysia, Malaysia.

Tilahun, S.L. (2017) 'Prey-predator hyperheuristic', Applied Soft Computing, Vol. 59, pp.104-114 [online] https://doi.org/10.1016/j.asoc.2017.04.044. 
Yang, X.S. (2011) 'Metaheuristic optimization: algorithm analysis and open problems', Experimental Algorithms, pp.21-32.

\section{Appendix}

\section{Datasets}

The dataset has three parts. The first one represented by variable $I$ contain the location of the centres in the coordinate system where the industry is located at the origin. Based on that the matrix $d$ represents the distance between the centres, i.e., $d_{i j}$ is the distance between centre $i$ and centre $j$. The vector $z$ is the vector which gives the distance of each centre from the industry.

\section{Dataset 1}

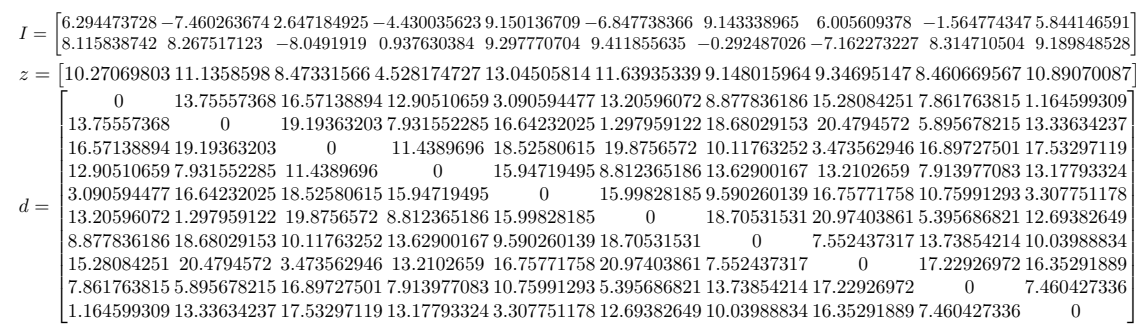

\section{Dataset 2}

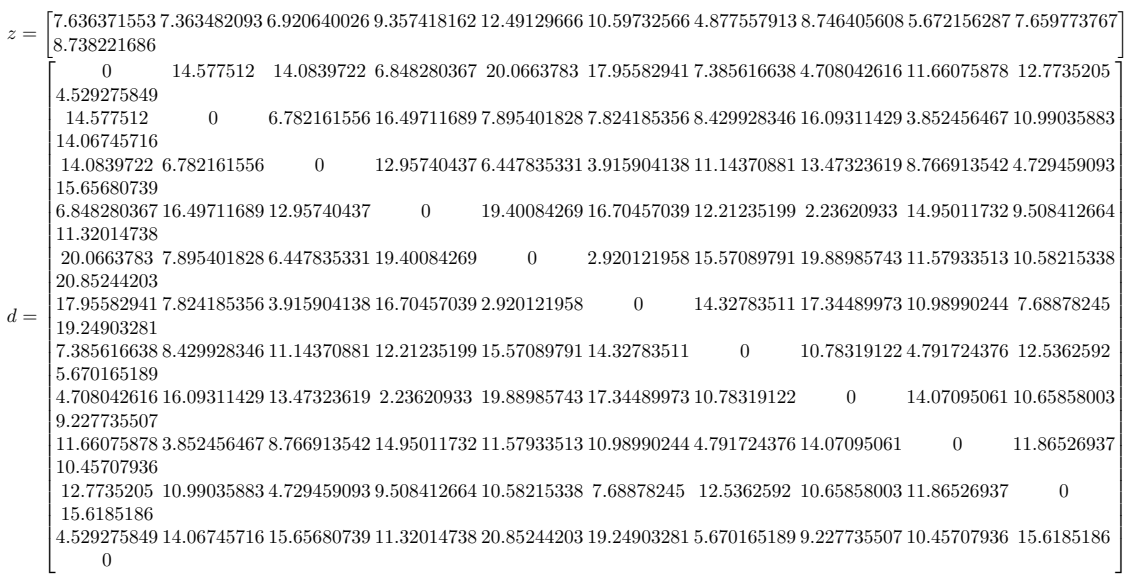

\section{Dataset 3}

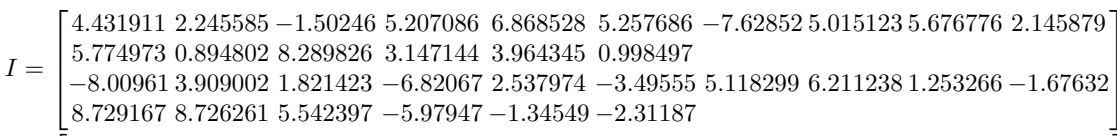

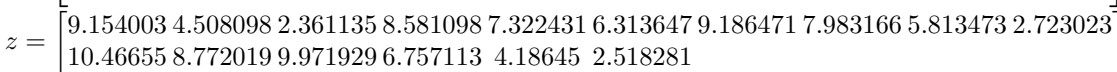




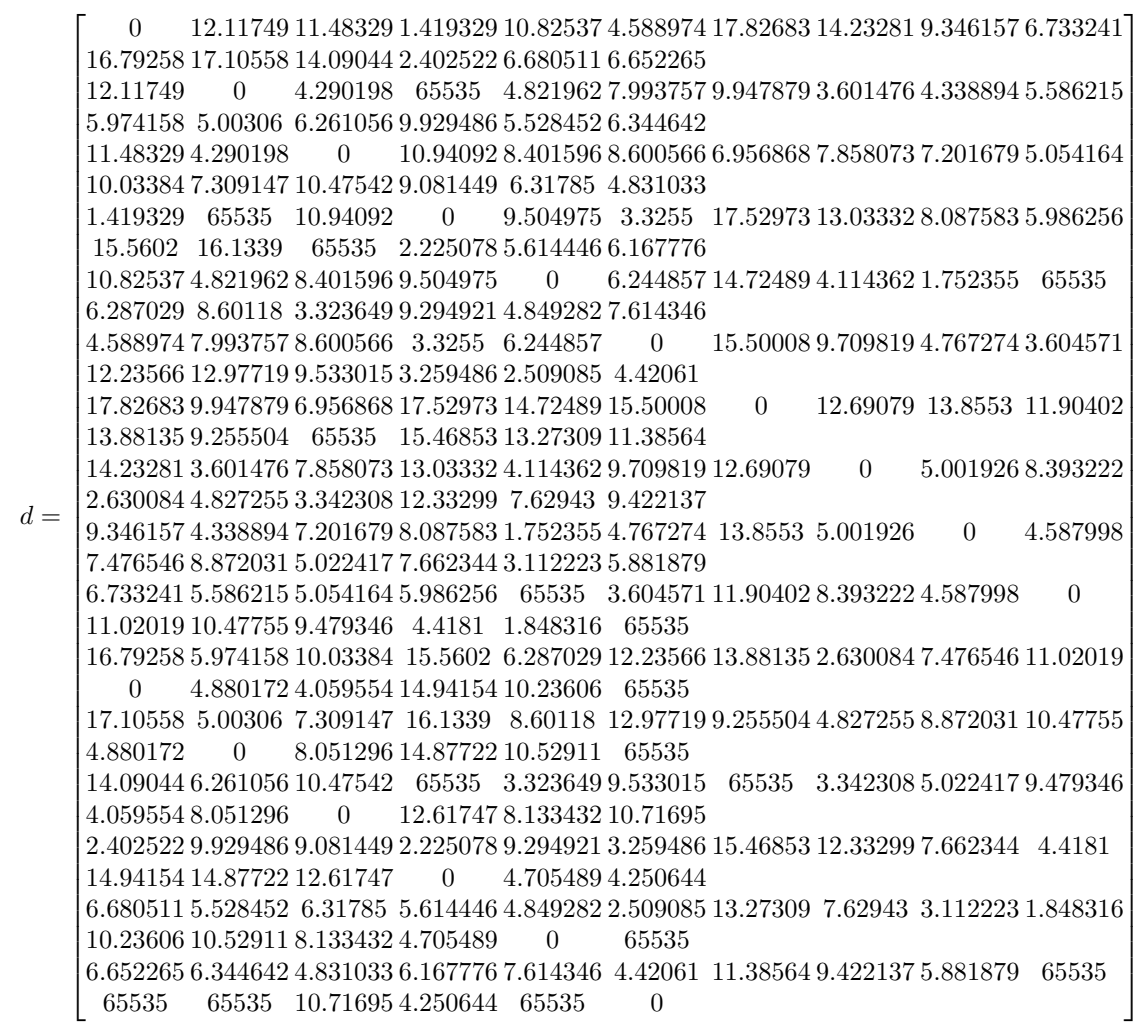

